\title{
Constraints on the NMSSM from the Oblique Parameters
}

\author{
Markos Maniatis and York Schröder \\ Fakultät für Physik, Universität Bielefeld, 33615 Bielefeld, Germany \\ Correspondence should be addressed to Markos Maniatis; maniatis@physik.uni-bielefeld.de
}

Received 10 December 2012; Accepted 2 February 2013

Academic Editor: Kingman Cheung

Copyright (C) 2013 M. Maniatis and Y. Schröder. This is an open access article distributed under the Creative Commons Attribution License, which permits unrestricted use, distribution, and reproduction in any medium, provided the original work is properly cited.

Electroweak precision measurements, encoded in the oblique parameters, give strong constraints on physics beyond the Standard Model. The oblique parameters $S, T, U(V, W, X)$ are calculated in the next-to-minimal supersymmetric model (NMSSM). We outline the calculation of the oblique parameters in terms of one-loop gauge-boson self-energies and find sensitive restrictions for the NMSSM parameter space.

\section{Introduction}

The precision measurements of the electroweak parameters give stringent constraints on physics beyond the Standard Model (SM). A very elegant method to systematically confront the electroweak precision measurements with new physics is given by the oblique parameters $S, T, U[1-3]$. These three parameters allow for restricting any physics beyond the $\mathrm{SM}$, under the following three conditions.

(i) The physics beyond the SM has to obey $S U(2)_{L} \otimes$ $U(1)_{Y}$ gauge symmetry; that is, there are no additional electroweak gauge bosons compared to the SM.

(ii) The couplings of new particles to light fermions have to be suppressed. That is, the main contribution of couplings beyond the SM to four-fermion scattering originates from the change in the self-energies of the gauge-boson propagators. These contributions are called oblique corrections. The suppressed contributions which may for instance appear in box diagrams with four external fermions or in vertex corrections are called nonoblique corrections.

(iii) New physics enters only at a large scale compared to the electroweak scale.

From the second condition it is clear that the oblique parameters are expressed in terms of gauge-boson self-energies, as shown in detail in [3]. The main argument is that the electroweak precision measurements probe weak-interaction processes with light external fermions of mass $m_{f}$ (at $\mathrm{cms}$ energies on the electroweak scale), wherein vertex- and boxtype corrections are suppressed by factors of $m_{f}^{2} / m_{Z}^{2}$ as compared to the self-energy loop corrections.

However, many models beyond the SM are expected to have effects at a scale not too far from the electroweak scale, which is given by the vacuum-expectation value of the neutral SM Higgs boson component $v_{0} \approx 174 \mathrm{GeV}$. In order to weaken the third condition for the oblique parameters $S, T, U$ mentioned previously, allowing new physics to enter already at a scale somewhat larger than the electroweak scale, the oblique parameters were extended to the six parameters $S, T$, $U, V, W, X[4,5]$. The explicit expressions for these oblique parameters read

$$
\begin{gathered}
S=\frac{4 s_{W}^{2} c_{W}^{2}}{\alpha}\left[\frac{\Pi_{Z Z}\left(m_{Z}^{2}\right)-\Pi_{Z Z}(0)}{m_{Z}^{2}}\right. \\
\left.-\frac{c_{W}^{2}-s_{W}^{2}}{s_{W} c_{W}} \Pi_{Z \gamma}^{\prime}(0)-\Pi_{\gamma \gamma}^{\prime}(0)\right], \\
T=\frac{1}{\alpha}\left[\frac{\Pi_{W W}(0)}{m_{W}^{2}}-\frac{\Pi_{Z Z}(0)}{m_{Z}^{2}}\right],
\end{gathered}
$$




$$
\begin{aligned}
U=\frac{4 s_{W}^{2}}{\alpha}[ & \frac{\Pi_{W W}\left(m_{W}^{2}\right)-\Pi_{W W}(0)}{m_{W}^{2}} \\
& -c_{W}^{2} \frac{\Pi_{Z Z}\left(m_{Z}^{2}\right)-\Pi_{Z Z}(0)}{m_{Z}^{2}} \\
& \left.-2 s_{W} c_{W} \Pi_{Z \gamma}^{\prime}(0)-s_{W}^{2} \Pi_{\gamma \gamma}^{\prime}(0)\right], \\
V=\frac{1}{\alpha} & {\left[\Pi_{Z Z}^{\prime}\left(m_{Z}^{2}\right)-\frac{\Pi_{Z Z}\left(m_{Z}^{2}\right)-\Pi_{Z Z}(0)}{m_{Z}^{2}}\right], } \\
W=\frac{1}{\alpha} & {\left[\Pi_{W W}^{\prime}\left(m_{W}^{2}\right)-\frac{\Pi_{W W}\left(m_{W}^{2}\right)-\Pi_{W W}(0)}{m_{W}^{2}}\right], } \\
X=- & \frac{s_{W} c_{W}}{\alpha}\left[\frac{\Pi_{Z \gamma}\left(m_{Z}^{2}\right)}{m_{Z}^{2}}-\Pi_{Z \gamma}^{\prime}(0)\right]
\end{aligned}
$$

The quantities $\Pi_{G_{1} G_{2}}(s)$ with $G_{1 / 2} \in\{\gamma, W, Z\}$ denote the new contributions to the transverse part of the self-energies at a momentum-squared scale $s$ compared to the SM,

$$
\Pi_{G_{1} G_{2}}(s)=\Pi_{G_{1} G_{2}}^{\text {new }}(s)-\Pi_{G_{1} G_{2}}^{\mathrm{SM}}(s) .
$$

The derivatives of the self-energies $\Pi_{G_{1} G_{2}}(s)$ with respect to the scale $s$ are denoted by $\Pi_{G_{1} G_{2}}^{\prime}\left(s_{0}\right)=\mathrm{d} \Pi_{G_{1} G_{2}}(s) /\left.\mathrm{d} s\right|_{s=s_{0}}$. The fact that only relatively few parameters (besides $\Pi(s)$ for $s \in$ $\left\{0, m_{W}^{2}, m_{Z}^{2}\right\}$ only $\Pi^{\prime}(s)$ at the same low-energy scales) enter in (2) reflects the observation that precision measurements are made only by two-particle scatterings on light fermions at those few scales, as explained in detail in [4]. Finally, $s_{W}=$ $\sin \left(\theta_{W}\right)$ and $c_{W}=\cos \left(\theta_{W}\right)$ contain the usual weak Weinberg mixing angle $\theta_{W}$, and $\alpha$ denotes the fine-structure constant.

Having defined the oblique parameters, electroweak precision observables, like for instance the $W^{ \pm}$-boson mass, may be expressed in terms of these parameters. Constraints on the oblique parameters are gained via a global fit to the electroweak precision measurements; see for example, [6]. Being exactly zero within the SM, these global fits result in error bands for the six parameters of (1), see (3), hence potentially constraining the size of effects from new physics.

In this paper, we compute the oblique parameters $S, T$, $U, V, W, X$ of (1) in the next-to-minimal supersymmetric extension of the SM (NMSSM); for reviews of the NMSSM, we refer to $[7,8]$. Let us briefly recall that in the superpotential of the NMSSM the $\mu$-term of the MSSM is replaced by a $\lambda$ term and a $\kappa$ term. In contrast to the parameter $\mu$, the new parameters $\lambda$ and $\kappa$ are dimensionless; in particular a vacuum-expectation value of a Higgs singlet times $\lambda$ generates an effective $\mu$ term, and the $\kappa$ term avoids a PecceiQuinn symmetry.

The NMSSM has recently received much attention; in particular, besides the scale invariant superpotential, it has a much richer Higgs sector and a fifth neutralino compared to the minimal supersymmetric extension (MSSM). Noting that in the fermion-fermion interactions there appear in principle also nonoblique corrections in the NMSSM, here we assume that the nonoblique corrections are negligible.

There are several computations of electroweak precision observables in the NMSSM, of which we would like to mention a few here. First, in a study of the $Z^{0}$ boson width [9], supposing that the lightest neutralino $\widetilde{\chi}_{1}^{0}$ in the NMSSM has a mass $m_{\tilde{\chi}_{1}^{0}}<m_{Z} / 2$, it has been shown that the Z-boson decay width changes as compared to the SM and hence a comparison with precision measurements (in particular at LEP) gives strong constraints on the NMSSM parameters. Second, in [10], the partial decay of $Z$ bosons into $b$ quark pairs has been considered. Here it is found via a parameter scan that the $Z \bar{b} b$ coupling in the SM compared to data can not be significantly improved in the MSSM/NMSSM. Third, the new contributions of the NMSSM to the transverse parts of the $W^{ \pm}$- and the $Z$-boson selfenergies have been presented in [11]. Fourth, leptonic decays of the $Z$ as well as $W^{ \pm}$bosons in the NMSSM were considered in [12]. In particular, in this way, constraints on the couplings respectively masses of the new particles, which appear in the loops, have been obtained.

Since the parameter space of the NMSSM is very large, there are different approaches to phenomenological studies of this model. In [13-15], for instance, the constrained version of the NMSSM is considered where it is assumed that various masses and couplings unify at the GUT scale. Another approach is to consider specific benchmarks scenarios, representing different regimes in parameter space $[16,17]$. In our numerical examples mentioned later, we will adopt the former approach. Let us remark that there exist similar approaches of electroweak precision observables in the MSSM; see for instance [18-21].

\section{Details of the Calculation}

For the prediction of the oblique parameters of (1) we need to compute the transverse parts of the one-loop self-energies $\Pi_{G_{1} G_{2}}(s)=\Pi_{G_{1} G_{2}}^{\mathrm{NMSSM}}(s)-\Pi_{G_{1} G_{2}}^{\mathrm{SM}}(s)$, where $G_{1}, G_{2}$ denote the gauge bosons $\gamma, W^{ \pm}, Z^{0}$. The self-energies with exclusively leptons, quarks, and gauge bosons in the loops are exactly the same in $\Pi_{G_{1} G_{2}}^{\mathrm{NMSSM}}(s)$ and $\Pi_{G_{1} G_{2}}^{\mathrm{SM}}(s)$ and therefore do not need to be evaluated. As a consistency check, however, we confirmed this analytically.

Since the Higgs sector of the NMSSM is not a simple extension of the SM Higgs sector, we have to consider in $\Pi_{G_{1} G_{2}}^{\mathrm{SM}}(s)$ all contributions which contain the SM Higgs boson $H_{\text {SM }}$. In the self-energies of the NMSSM we have to consider all contributions which involve scalar neutrinos $\tilde{v}$, scalar leptons $\widetilde{l}$, scalar up- and down-type quarks $\widetilde{u}, \widetilde{d}$, neutralinos $\chi^{0}$, charginos $\chi^{+}$, the neutral Higgs bosons $H_{1}, H_{2}, H_{3}, A_{1}$, $A_{2}$, the pair of charged Higgs bosons $H^{ \pm}$, and the Goldstone bosons $G^{0}, G^{+}$. All Feynman diagrams of the self-energy contributions to the oblique parameters are shown in the appendix. Let us note that we consider the most general NMSSM in our computation. In particular we allow for CP violation in the Higgs sector, such that the neutral Higgs 
TABLE 1: Parameter values for studies in case of the constrained NMSSM $\left(M_{i}\right.$ and $A_{i}$ in GeV). These sets are inspired by the ranges given in Figures $1-3$ of [15].

\begin{tabular}{lcccccc}
\hline Set \# & $M_{0}^{\mathrm{GUT}}$ & $M_{1 / 2}^{\mathrm{GUT}}$ & $A_{0}^{\mathrm{GUT}}$ & $A_{\kappa}^{\mathrm{GUT}}$ & $\tan (\beta)^{\mathrm{MSUSY}}$ & $\operatorname{sgn}(\mu)$ \\
\hline 1 & 500 & 500 & -800 & -100 & 5 & + \\
2 & 500 & 500 & -800 & -1500 & 1.7 & +15 \\
3 & 100 & 200 & -700 & -75 & 5 & + \\
\hline
\end{tabular}

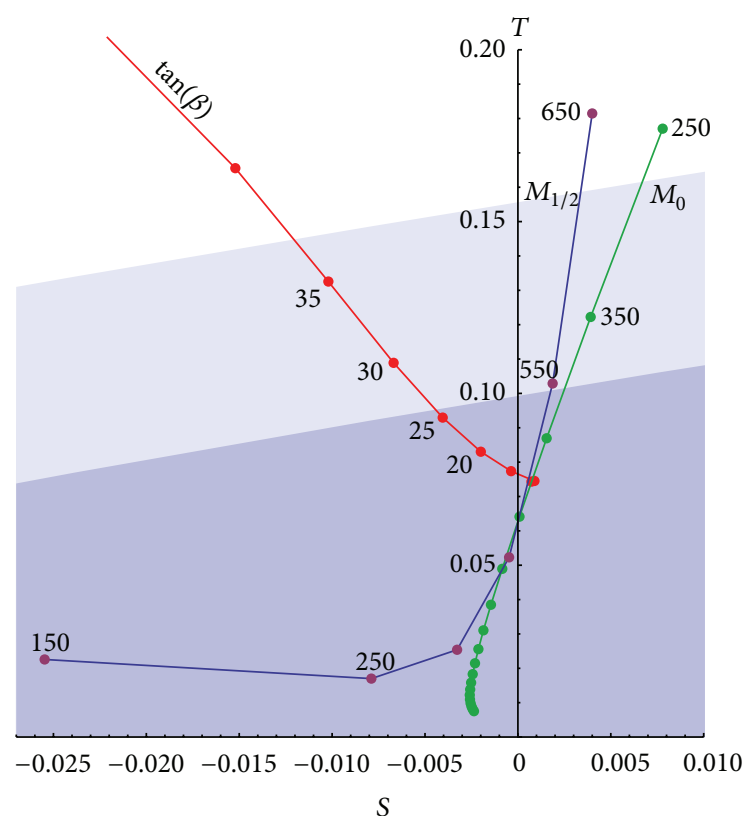

FIgURE 1: Oblique parameters $S$ and $T$ in the NMSSM in the constrained case with central parameters $\tan (\beta)=5, M_{0}=M_{1 / 2}=$ $500 \mathrm{GeV}, A_{0}=-800 \mathrm{GeV}, A_{\kappa}=-100 \mathrm{GeV}, \operatorname{sgn}(\mu)=+$, and $\lambda=0.15$ (first row of Table 1) with variation of the parameters successively in the ranges $\tan (\beta)=5,10, \ldots, 40(\tan (\beta)=5,10$ lie very close together), $M_{0}=250 \mathrm{GeV}, 350 \mathrm{GeV}, \ldots, 1950 \mathrm{GeV}, M_{1 / 2}=150 \mathrm{GeV}$, $250 \mathrm{GeV}, \ldots, 650 \mathrm{GeV}$ as indicated in the figure. The shaded regions show the $1 \sigma$ and $2 \sigma$ error ellipses of the electroweak precision measurements fitted to $S$ and $T$ corresponding to (3) [6].

bosons $H_{i} / A_{j}$ are not necessarily $\mathrm{CP}$ even/odd, respectively; for details see for instance [7].

The NMSSM Feynman rules are implemented in the FeynRules program package $[22,23]$ following the conventions of [24]. As a caveat, let us remark here that the Goldstone components of the neutral Higgs boson squared mixing matrix have to be carefully constructed to guarantee unitarity, which is violated by the parameters chosen in the model file nmssmfr. We link this list of Feynman rules with the packages FeynArts/FormCalc [25], resulting in analytic expressions for the various one-loop self-energies in terms of basic scalar master integrals. Next, we assemble the parameters of (2) and numerically evaluate the results using the program package LoopTools [26]. We observe that all ultraviolet singularities cancel between the different selfenergies in the oblique parameters. On a more technical note, the matrix $\gamma^{5}$ is treated naively (i.e., anticommuting) with $\left(\gamma^{5}\right)^{2}=\mathbb{1}_{4 \times 4}$, while we have checked explicit gauge parameter independence.

\section{Results}

First let us note that we expect in general different results for the oblique parameters in the NMSSM compared to that of the MSSM: on the one hand the (complex) Higgs singlet, introduced in the NMSSM, gives two more physical Higgs bosons compared to the MSSM which show up in the gauge-boson self-energies. On the other hand the additional singlino mixes, even that it is a gauge singlet, with the other neutralinos and therefore gives changed contributions. In general only in the limit of arbitrary small parameters $\lambda$ and $\kappa$ keeping the ratio $\kappa / \lambda$ as well as the product $\lambda v_{s}$ fixed (with $v_{s}$ the vacuum-expectation value of the singlet) and keeping also the trilinear couplings $A_{\lambda}$ and $A_{\kappa}$ fixed, the NMSSM becomes the MSSM.

As a simple numerical example, we assume unification of scalar masses $M_{0}$, fermion masses $M_{1 / 2}$, and trilinear couplings $A_{0}$. This scenario is usually called constrained NMSSM (cNMSSM). To be precise, we employ the program package NMSPEC [15], with a unification of the scalars, fermions, and trilinear couplings but with the exception of the singlet mass $m_{s}$ and the Peccei-Quinn parameter $\kappa$, which are given as output parameters, determined by the minimization equations of the Higgs potential. In addition $A_{\kappa}$ is not unified with the other trilinear couplings and is varied separately. Furthermore, the ratio of the vacuumexpectation value of the two Higgs boson doublets, $\tan (\beta)$, the Higgs coupling parameter $\lambda$, and the sign of $\mu$ have to be fixed in addition to the parameters of the SM. The program package NMSPEC eventually computes the mass spectra and mixing angles at the electroweak scale. Let us note that our calculation of the oblique parameters is performed in the general NMSSM such that the oblique parameters for arbitrary parameter values can be easily computed. The program code for the oblique parameters is available as $\mathrm{C}$-code from the URL [27].

The explicit values for the NMSSM parameters we choose in our numerical examples are given in Table 1. These parameter sets are inferred from the figures presented in [15]. The scales at which the NMSSM parameters are fixed are written as a superscript, with MSUSY and GUT the supersymmetry breaking scale, respectively, the grand unification scale, respectively; both scales are derived from the input parameters in NMSPEC. We note that the chosen parameter sets as given in Table 1 pass all the different constraints, gained from Higgs boson searches, partial decay width of the $Z$ 


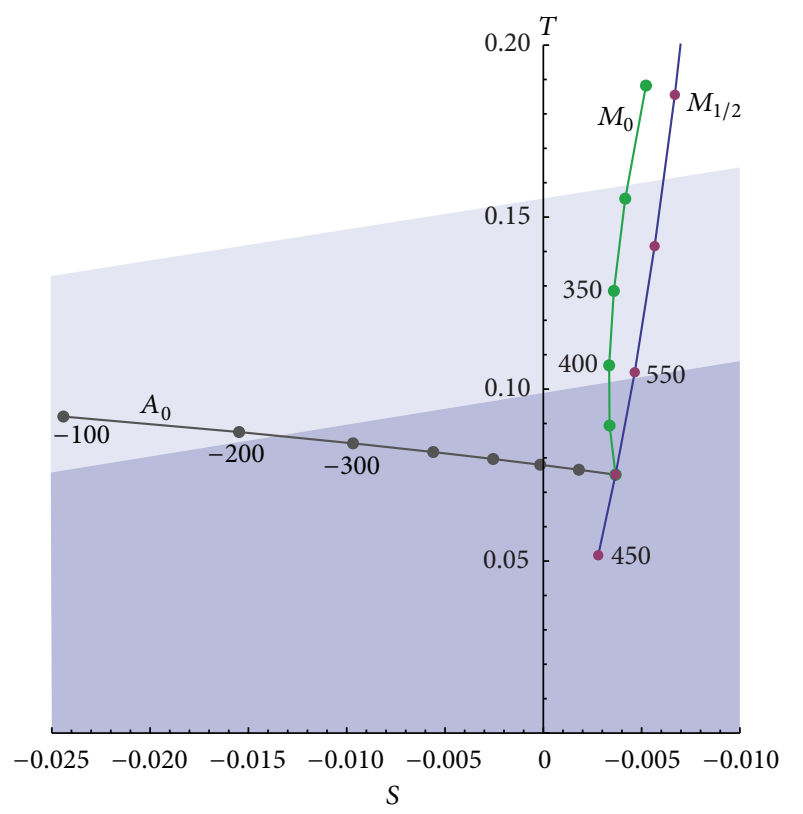

(a)

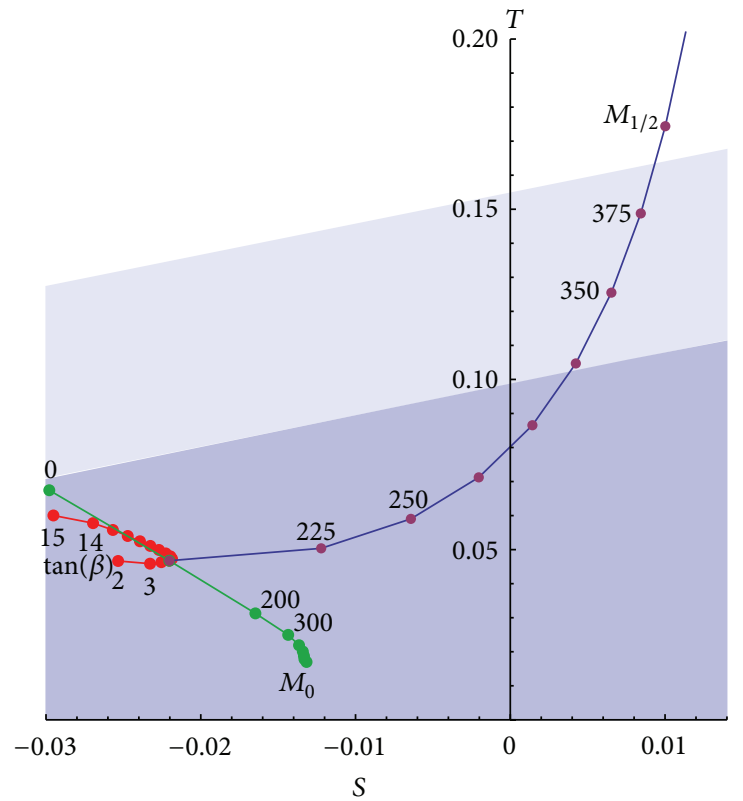

(b)

FIGURE 2: (a): Oblique parameters $S$ and $T$ in the NMSSM in the constrained case with central parameters $\tan (\beta)=1.7, M_{0}=M_{1 / 2}=500 \mathrm{GeV}$, $A_{0}=-800 \mathrm{GeV}, A_{\kappa}=-1500 \mathrm{GeV}, \operatorname{sgn}(\mu)=+$, and $\lambda=0.5$ (second row in Table 1) with successive variations of the parameters $M_{0}=$ $250 \mathrm{GeV}, 300 \mathrm{GeV}, \ldots, 500 \mathrm{GeV}, M_{1 / 2}=450 \mathrm{GeV}, 500 \mathrm{GeV}, \ldots, 650 \mathrm{GeV}, A_{0}=-800 \mathrm{GeV},-700 \mathrm{GeV}, \ldots,-100 \mathrm{GeV}$, as indicated in the figure. (b): Same as (a) but with central parameters $\tan (\beta)=5, M_{0}=100 \mathrm{GeV}, M_{1 / 2}=200 \mathrm{GeV}, A_{0}=-700 \mathrm{GeV}, A_{\kappa}=-75 \mathrm{GeV}$, $\operatorname{sgn}(\mu)=+$, and $\lambda=0.2$ with successive variations of the parameters $\tan (\beta)=2,3, \ldots, 15, M_{0}=0 \mathrm{GeV}, 100 \mathrm{GeV}, \ldots, 800 \mathrm{GeV}, M_{1 / 2}=200 \mathrm{GeV}, 225 \mathrm{GeV}, \ldots, 400 \mathrm{GeV}$.
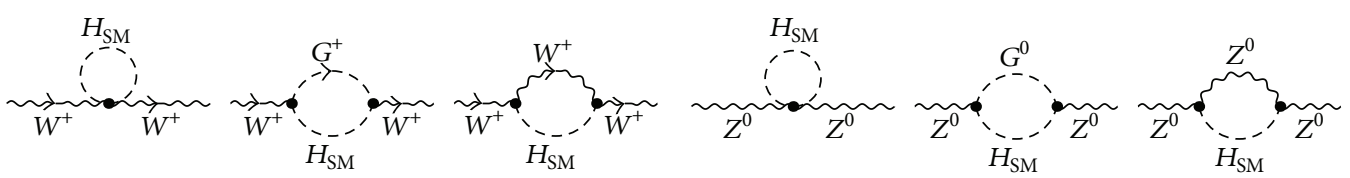

FIGURE 3: Feynman diagrams for the self-energies $\Pi_{W W}^{S M}(s)$ and $\Pi_{Z Z}^{S M}(s)$ which contribute to the oblique parameters. All other diagrams vanish, respectively, cancel with the corresponding diagrams in $\Pi_{W W}^{\mathrm{NMSSM}}(s)$ and $\Pi_{Z Z}^{\mathrm{NMSSM}}(s)$.

boson into neutralinos, and mass bounds for the charginos; for further details we refer to [15].

In Figures 1 and 2 we present the results for the oblique parameters $S$ and $T$ for the different parameter sets given in Table 1. All other oblique parameters turn out to be rather small and are therefore not shown explicitly. In the Figures we vary successively the parameters $A_{0}, \tan (\beta), M_{0}$, and $M_{1 / 2}$ about the central values from Table 1 as indicated in the figures (where we suppress the superscripts MSUSY and GUT). From the lines we see how the oblique parameters $S$ and $T$ change under variations of the parameter values. We also draw the $1 \sigma$ and $2 \sigma$ error ellipses corresponding to the recent experimental fits to $S$ and $T$ [6]:

$$
S=0.01 \pm 0.1, \quad T=0.03 \pm 0.11, \quad \rho=0.87 .
$$

Here, $\rho$ denotes the correlation coefficient. Note that in this fit a SM Higgs boson mass of $m_{H_{\mathrm{SM}}}=117 \mathrm{GeV}$ is assumed, which we also use consistently as a parameter value in the SM self-energies.
As expected, in our numerical examples we find suppressed contributions to the oblique parameters $V, W, X$, which is due to the large masses of the additional particles as compared to the electroweak scale. The sensitivity of the oblique parameters under variations of NMSSM parameters is clearly visible: for the central parameter set 1 in Table 1 . We infer from Figure 1 that the $2 \sigma$ error ellipse constrains $\tan (\beta) \lesssim 40, M_{0} \gtrsim 250 \mathrm{GeV}$, and $M_{1 / 2} \lesssim 650 \mathrm{GeV}$. For the other central values in Table 1 we can easily read off the constraints from Figure 2. In general the oblique parameters $S$ and $T$ are highly sensitive to new particles which are nonisosinglets. However, only under certain assumptions the origin of the contributions to the different oblique parameters is immediately evident; we refer to the discussion in [3] for further details. Since these assumptions are not fulfilled in our case we refrain from a detailed study here. It would be also interesting to discuss the impact of the NMSSM parameters at the electroweak scale on the oblique parameters. However, this is beyond the scope of the present paper, where we focus on the calculation of the oblique parameters itself. 


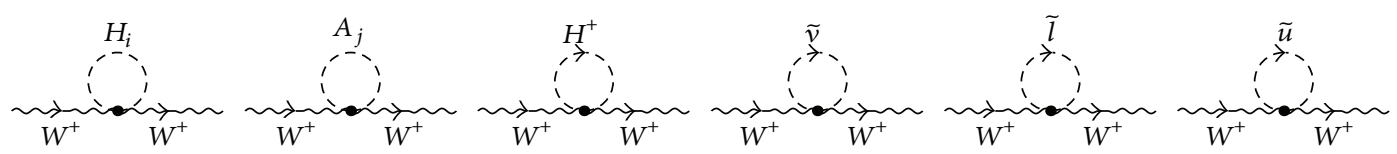

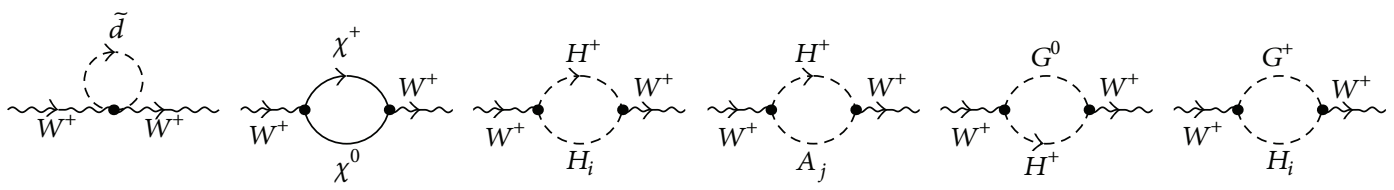

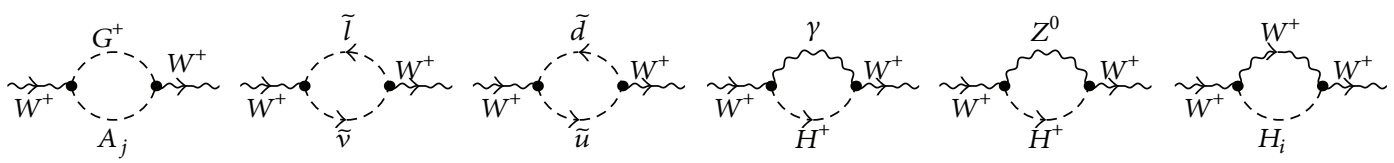

FIGURE 4: Feynman diagram contribution to the self-energy $\Pi_{W W}^{\mathrm{NMSSM}}(s)$.

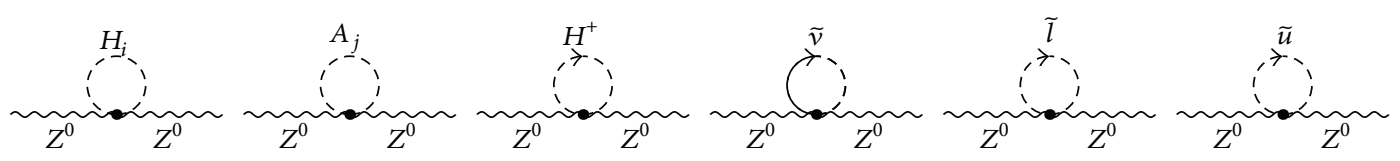

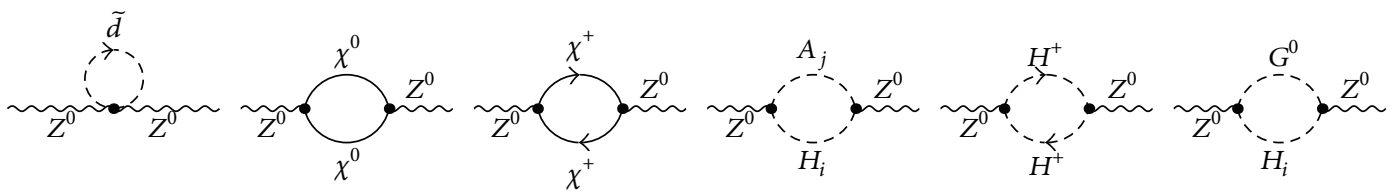

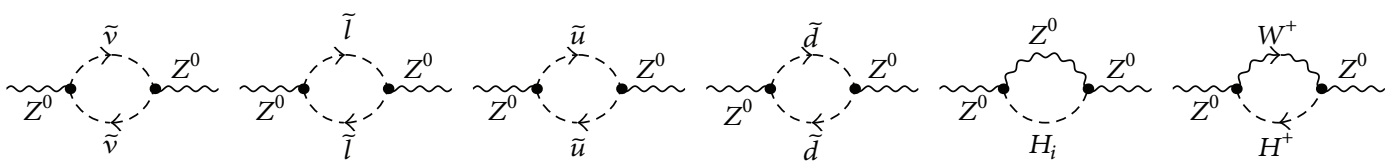

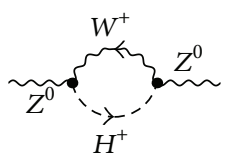

Figure 5: Feynman diagram contribution to the self-energy $\Pi_{Z Z}^{\text {NMSSM }}(s)$.

\section{Conclusions}

For a large class of models beyond the Standard Model, the so-called oblique parameters give very sensitive constraints coming from electroweak precision measurements. We have computed the set of extended oblique parameters $S, T, U$, $V, W, X$ for the next-to-minimal supersymmetric model (NMSSM).

We have presented numerical examples with the parameters of the NMSSM chosen in a constrained case, as explained in Section 3. We observe the oblique parameters $S$ and $T$ to be highly sensitive on variations of the model parameters. In fact, fairly modest changes of the NMSSM parameters easily violate the constraints from the electroweak precision measurements.

The oblique parameters have been computed for the general case, in particular with a general CP violating Higgs sector, such that they may be applied to arbitrary parameter values, in a more complete parameter scan, which we reserve for future work.

\section{Appendix}

\section{Feynman Diagrams for the Oblique Parameters}

Here we present the Feynman diagrams which contribute to the oblique parameters of (1). For self-energy diagrams which exclusively have leptons, quarks, and gauge bosons in the loops, the contributions to $\Pi_{G_{1} G_{2}}^{\mathrm{NMSSM}}(s)$ and $\Pi_{G_{1} G_{2}}^{\mathrm{SM}}(s)$ exactly are canceled in (2) and do not have to be computed.

The contributions to $\Pi_{G_{1} G_{2}}^{\mathrm{SM}}(s)$ consist of diagrams which contain the SM Higgs boson $\left(H_{\mathrm{SM}}\right)$ in the loop. There are only contributions of this kind to the $W^{+}$and $Z^{0}$ self-energies as shown in Figure 3.

We also show all self-energy diagrams contributing to the NMSSM part of the oblique parameters. These diagrams involve scalar neutrinos $\widetilde{v}$, scalar leptons $\tilde{l}$, scalar up- and down-type quarks $\tilde{u}, \widetilde{d}$, neutralinos $\chi^{0}$, and charginos $\chi^{+}$, as well as the neutral Higgs bosons $H_{i}, A_{j}$, the charged Higgs 


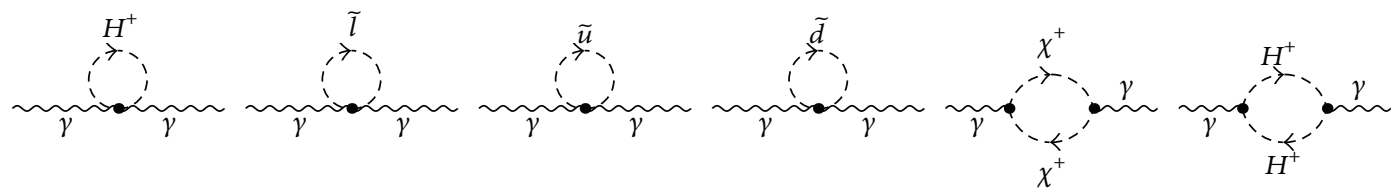

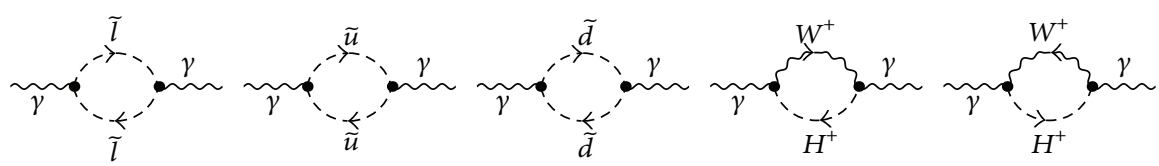

FIGURE 6: Feynman diagram contribution to the self-energy $\Pi_{\gamma \gamma}^{\mathrm{NMSSM}}(s)$.
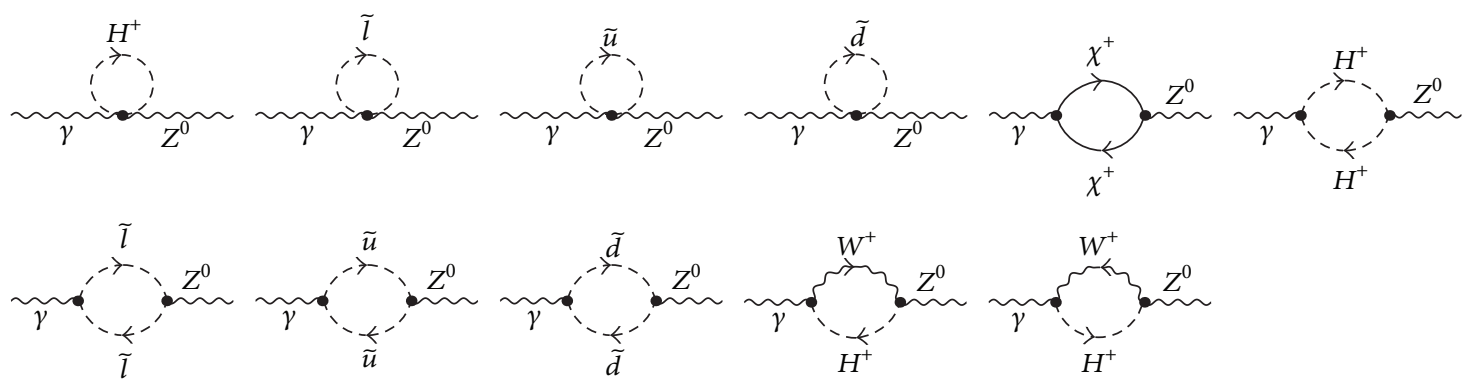

FIGURE 7: Feynman diagram contribution to the self-energy $\Pi_{Z \gamma}^{\mathrm{NMSSM}}(s)$.

bosons $H^{ \pm}$, and the Goldstone bosons $G^{0}, G^{+}$. All other contributions, for instance, the self-energy with a lepton loop, are canceled with the corresponding SM contribution.

The $W^{+}, Z^{0}$, photon, $Z^{0}$-photon self-energy diagrams are shown in Figures 4, 5, 6, and 7, respectively.

\section{Acknowledgments}

The authors are grateful to B. Fuks for quick response concerning an update of the NMSSM model file contained in FeynRules. The work of Y. Schröder is supported by the Heisenberg Program of the Deutsche Forschungsgemeinschaft (DFG), Contract no. SCHR 993/1.

\section{References}

[1] M. E. Peskin and T. Takeuchi, "New constraint on a strongly interacting Higgs sector," Physical Review Letters, vol. 65, no. 8, pp. $964-967,1990$.

[2] D. C. Kennedy and P. Langacker, "Precision electroweak experiments and heavy physics: a global analysis," Physical Review Letters, vol. 65, no. 24, pp. 2967-2970, 1990, Erratum-ibid. vol. 66, p. 395, 1991.

[3] M. E. Peskin and T. Takeuchi, "Estimation of oblique electroweak corrections," Physical Review D, vol. 46, no. 1, pp. 381409, 1992.

[4] I. Maksymyk, C. P. Burgess, and D. London, "Beyond S, T and U," Physical Review D, vol. 50, no. 1, pp. 529-535, 1994.

[5] C. P. Burgess, S. Godfrey, H. König, D. London, and I. Maksymyk, "A global fit to extended oblique parameters," Physics Letters B, vol. 326, no. 3-4, pp. 276-281, 1994.
[6] J. Beringer, J. F. Arguin, R. M. Barnett et al., "Review of Particle Physics (RPP)," Physical Review D, vol. 86, Article ID 010001, 2012.

[7] M. Maniatis, “The next-to-minimal supersymmetric extension of the standard model reviewed," International Journal of Modern Physics A, vol. 25, no. 18, pp. 3505-3602, 2010.

[8] U. Ellwanger, C. Hugonie, and A. M. Teixeira, "The next-tominimal supersymmetric standard model," Physics Reports, vol. 496, no. 1-2, pp. 1-77, 2010.

[9] D. Teyssier, "A flavour-independent search for a hadronically decaying neutral Higgs bosons at LEP with the L3 detector," International Journal of Modern Physics A, vol. 16, supplement 1, pp. 819-821, 2001.

[10] J. Cao and J. M. Yang, "Anomaly of Zb antib coupling revisited in MSSM and NMSSM," Journal of High Energy Physics, vol. 12, article 006, 2008.

[11] G. Degrassi and P. Slavich, "On the radiative corrections to the neutral Higgs boson masses in the NMSSM," Nuclear Physics B, vol. 825, no. 1-2, pp. 119-150, 2010.

[12] F. Domingo and T. Lenz, "W mass and Leptonic Z-decays in the NMSSM," Journal of High Energy Physics, vol. 1107, p. 101, 2011, http://arxiv.org/abs/1101.4758.

[13] J. F. Gunion, Y. Jiang, and S. Kraml, "The constrained NMSSM and Higgs near 125 GeV,' Physics Letters B, vol. 710, pp. 454-459, 2012, http://arxiv.org/abs/1201.0982.

[14] A. Djouadi, M. Drees, U. Ellwanger et al., "Benchmark scenarios for the NMSSM," Journal of High Energy Physics, vol. 7, article $002,2008$.

[15] U. Ellwanger and C. Hugonie, "NMSPEC: a Fortran code for the sparticle and Higgs masses in the NMSSM with GUT scale boundary conditions," Computer Physics Communications, vol. 177, no. 4, pp. 399-407, 2007. 
[16] S. F. King, M. M. Mühlleitner, and R. Nevzorov, "NMSSM Higgs benchmarks near 125 GeV," Nuclear Physics B, vol. 860, p. 207, 2012, http://arxiv.org/abs/1201.2671.

[17] S. S. AbdusSalam, B. C. Allanach, H. K. Dreiner et al., "Benchmark models, planes, lines and points for future SUSY searches at the LHC," The European Physical Journal C, vol. 71, p. 1835, 2011.

[18] A. Dedes, A. B. Lahanas, and K. Tamvakis, "Effective weak mixing angle in the MSSM," Physical Review D, vol. 59, no. 1, Article ID 015019, 17 pages, 1999.

[19] S. Heinemeyer, W. Hollik, and G. Weiglein, "Electroweak precision observables in the minimal supersymmetric standard model," Physics Reports, vol. 425, no. 5-6, pp. 265-368, 2006.

[20] M. J. Ramsey-Musolf and S. Su, "Low energy precision test of supersymmetry," Physics Reports, vol. 456, pp. 1-88, 2008.

[21] G.-C. Cho, K. Hagiwara, Y. Matsumoto, and D. Nomura, "The MSSM confronts the precision electroweak data and the muon g-2," Journal of High Energy Physics, vol. 1111, article 068, 2011, http://arxiv.org/abs/1104.1769.

[22] N. D. Christensen and C. Duhr, "Feyn-rules-Feynman rules made easy," Computer Physics Communications, vol. 180, no. 9, pp. 1614-1641, 2009.

[23] C. Duhr and B. Fuks, "A superspace module for the Feyn-Rules package," Computer Physics Communications, vol. 182, no. 11, pp. 2404-2426, 2011.

[24] B. C. Allanach, C. Balazs, G. Belanger et al., "SUSY Les Houches Accord 2," Computer Physics Communications, vol. 180, no. 1, pp. 8-25, 2009.

[25] T. Hahn, "Generating Feynman diagrams and amplitudes with FeynArts 3," Computer Physics Communications, vol. 140, no. 3, pp. 418-431, 2001.

[26] T. Hahn and M. Perez-Victoria, "Automatized one loop calculations in four-dimensions and Ddimensions," Computer Physics Communications, vol. 118, no. 2-3, pp. 153-165, 1999.

[27] "C-code for the oblique parameters," http://www.physik.unibielefeld.de/theory/e6/BI-TP-2012-16.html. 

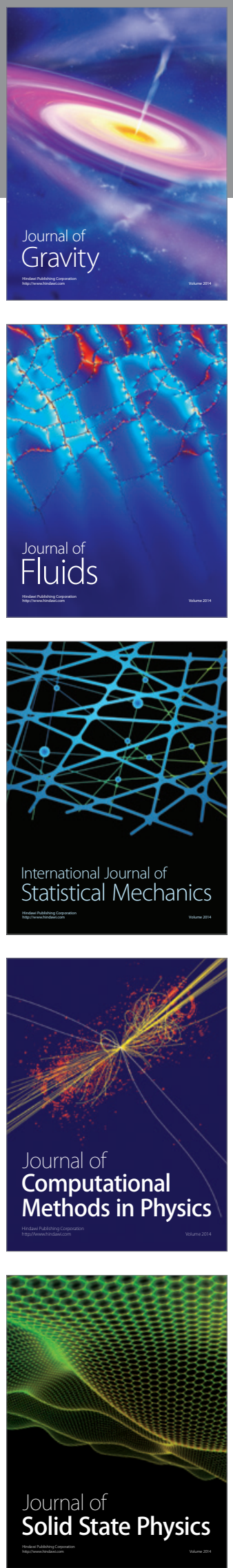

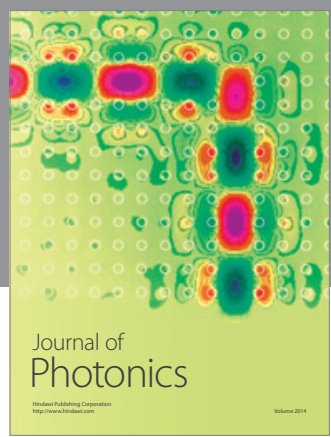

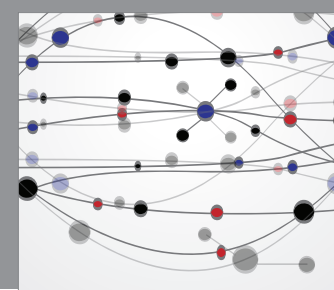

The Scientific World Journal

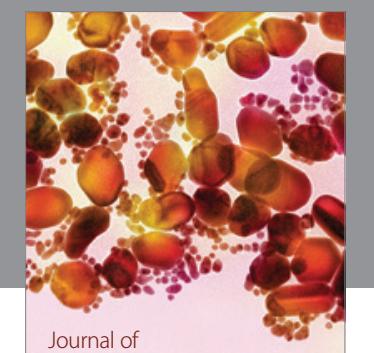

Soft Matter
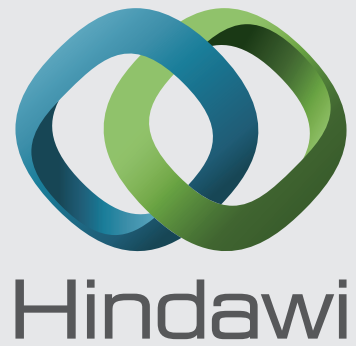

Submit your manuscripts at

http://www.hindawi.com
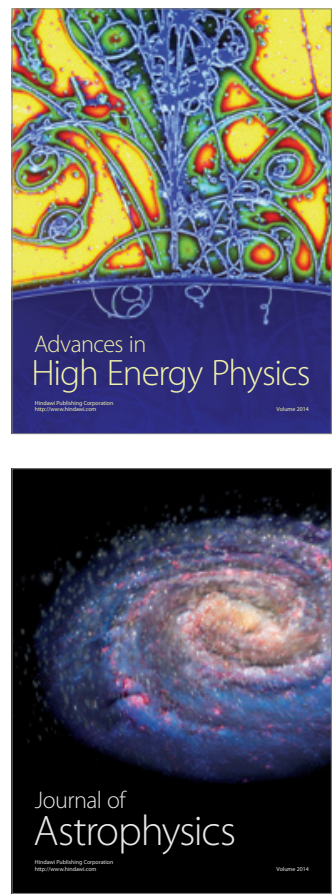
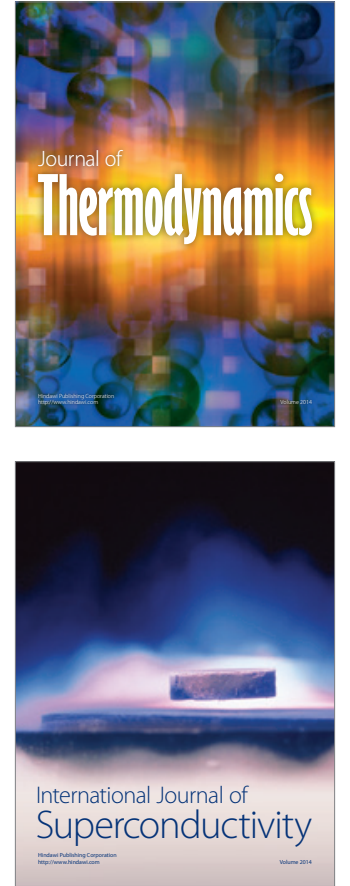
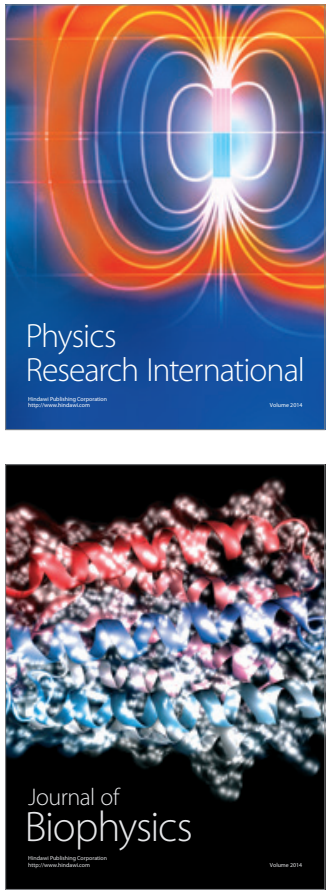
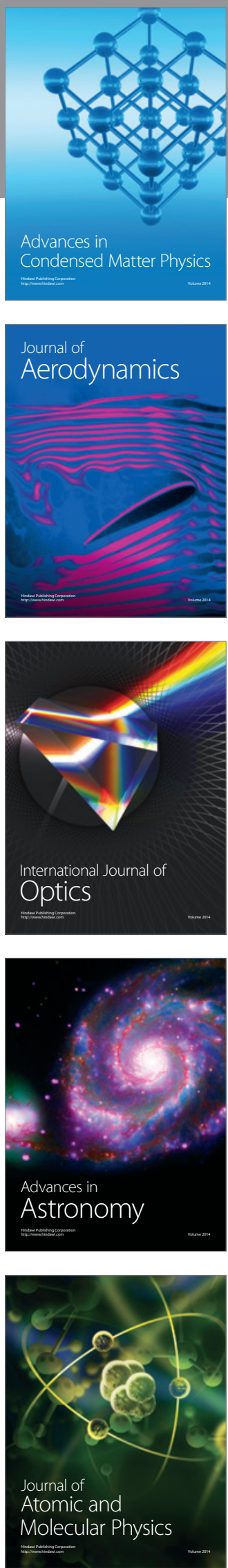